EPJ Web of Conferences 80, 00028 (2014)

DOI: $10.1051 /$ epj conf/20148000028

C) Owned by the authors, published by EDP Sciences, 2014

\title{
Overview of recent ALICE results
}

\author{
Roberta Arnaldi ${ }^{1}$,a for the ALICE Collaboration \\ ${ }^{1}$ INFN,Sezione di Torino
}

\begin{abstract}
ALICE is the LHC experiment devoted to the study of heavy-ion collisions. While results from $\mathrm{Pb}-\mathrm{Pb}$ collisions at $\sqrt{s_{\mathrm{NN}}}=2.76 \mathrm{TeV}$ provide insight on the properties of the plasma of quarks and gluons, formed in nucleus-nucleus interactions, the study of $\mathrm{p}-\mathrm{Pb}$ collisions at $\sqrt{s_{\mathrm{NN}}}=5.02 \mathrm{TeV}$ allows a deeper understanding of cold nuclear matter effects. Therefore, $\mathrm{p}-\mathrm{Pb}$ results turn out to be a powerful tool to provide a baseline for $\mathrm{Pb}-\mathrm{Pb}$, to correctly quantify how the various observables are affected by genuine hot medium effects. In this proceeding, a selection of the most recent ALICE results on the medium global properties and on heavy-flavour and quarkonium production will be discussed.
\end{abstract}

\section{Introduction}

Quantum ChromoDynamics (QCD) predicts, at high temperatures and/or baryonic density, a phase transition from the hadronic matter to a deconfined phase, where quarks and gluons are no more confined into nucleons. The conditions required for the formation of this new state of matter, named plasma of quarks and gluons (QGP), can be experimentally accessed in relativistic heavy-ion collisions.

ALICE (A Large Ion Collider Experiment) is one of the four large LHC experiments and its main objective is the study of the properties of the QGP. The ALICE experiment complements the experimental heavy-ion program, started about thirty years ago at the Brookhaven Alternating Gradient Synchrotron (AGS) and continued at the CERN Super Proton Synchrotron (SPS) up to $\sqrt{s_{N N}}=20 \mathrm{GeV}$ and finally at the Brookhaven Relativistic Heavy-Ion collider (RHIC) up to $\sqrt{s_{N N}}=200 \mathrm{GeV}$. In the last five years ALICE has collected a large wealth of data, studying $\mathrm{Pb}-\mathrm{Pb}$ collisions at $\sqrt{s_{\mathrm{NN}}}=2.76$ $\mathrm{TeV}$ and to complement its heavy-ion program, also p-A and pp interactions, to be used as references, have been investigated. In particular, since in the "simpler" p-A collision system the conditions which should lead to the formation of the QGP are not fullfilled, hot matter related effects are not expected to set in. Therefore, the study of the behaviour of several probes in the cold nuclear matter provides a fundamental reference to understand the $\mathrm{Pb}-\mathrm{Pb}$ results, where hot matter effects are present on top of the cold matter ones.

One way to investigate the global properties of the QCD medium is via the collective behaviour of the bulk of particles produced in the collisions. In the hot and strongly interacting medium collective behaviours can arise and flow of particles, generated by the presence of pressure gradients, can be

\footnotetext{
a e-mail: arnaldi@to.infn.it
} 
detected, providing informations on the degree of thermalization of the system, on the transport properties of the medium and on the size of the fireball. Another way to investigate the QGP medium is based on the so-called "hard-probes", i.e. probes produced in the initial scatterings and exposed to the full evolution of the medium. Among the hard-probes there are the high momentum partons, which fragment into jets, the heavy quarks (charm and beauty) observed as open heavy flavour hadrons and the quarkonium resonances $(\mathrm{J} / \psi, \Upsilon \ldots)$.

ALICE is a multi-purpose experiment, designed to investigate a large number of the observables relevant for the characterization of the medium. In particular, ALICE is equipped with detectors providing high precision tracking and excellent particle identification capabilities, fundamental tools to cope with the high multiplicity environement typical of the LHC heavy-ion collisions. Details on the ALICE apparatus can be found in [1].

\section{Global properties}

The matter created in $\mathrm{Pb}-\mathrm{Pb}$ collisions at the LHC has been measured to be hotter, longer lived and with a larger volume with respect to the fireball created at RHIC in lower energy collisions. More in details, identical boson interferometry indicates that the freeze-out volume is increased by a factor of two with respect to RHIC, while the lifetime of the fireball is $40 \%$ larger [2].

The fireball produced in the $\mathrm{Pb}-\mathrm{Pb}$ collisions can be characterized through the measurement of the "elliptic flow". The initial space anisotropy of the overlap region of the colliding nuclei, in particular in semi-central collisions, translates into an anisotropy in momentum space of the produced particles, due to the collective expansion under $\phi$-dependent pressure gradients. The elliptic flow is evaluated as the second coefficient $v_{2}$ extracted from the Fourier decomposition of the particle azimuthal distributions relative to the reaction plane. The $v_{2}$ parameter provides a measurement of the collective motion, accessing the properties of the deconfined medium. A $v_{2} \sim 0$ suggests isotropic particles emission with respect to the reaction plane, not keeping memory of the initial asymmetry, while a non-zero $v_{2}$ would indicate a non-isotropic particles emission on the azimuthal plane. ALICE has recently measured the elliptic flow of identified particles, as a function of their transverse momentum $\left(p_{\mathrm{T}}\right)$. In Fig. 1 the $v_{2}$ for several different particles species, including pions, kaons, anti-protons, the $\phi$ meson and multistrange baryons is shown, in two $\mathrm{Pb}-\mathrm{Pb}$ centrality classes (10-20\% and 30-40\%) [3]. The $v_{2}$ increases as a function of the transverse momentum, up to $3-4 \mathrm{GeV} / \mathrm{c}$. The mass ordering, clearly visible in the ALICE result, is qualitatively described by viscous hydrodynamics at low $p_{\mathrm{T}}\left(p_{\mathrm{T}}<2.5 \mathrm{GeV} / \mathrm{c}\right)$. At high $p_{\mathrm{T}}$ the $v_{2}$ seems to scale with the number of constituent quarks, causing in this way, a different trend for mesons and baryons as already observed at RHIC energy. However ALICE results indicate that such a scaling is violated by about $20 \%$ in the most central collisions. The $v_{2}$ of the $\phi$ meson is of particular interest to investigate at the same time the mass ordering and the baryon-meson grouping [3]. Below a $p_{\mathrm{T}}$ of $2.5 \mathrm{GeV} / \mathrm{c}$, the $\phi v_{2}$ follows the mass ordering, while at higher $p_{\mathrm{T}}$ its behaviour depends on the centrality of the collisions, being close to the $v_{2}$ of the proton in central collisions and close to the pion $v_{2}$ in mid-central collisions. This suggests that the mass of the particle, and not the number of its constituent quarks, is the main driver for the $v_{2}$ in central $\mathrm{Pb}-\mathrm{Pb}$ collisions.

Other recent ALICE results are based on two-particles correlations, a powerful tool to explore the particle production mechanism $[4,5]$. These studies are based on the distributions of the relative angles $\Delta \eta$ and $\Delta \phi$ between a pair formed by a "trigger" particle and an "associated" particle, in given $p_{\mathrm{T}}$ intervals. The most recent ALICE results in this field refer to the $\mathrm{p}-\mathrm{Pb}$ measurements.

Two-particle correlations in $\mathrm{p}-\mathrm{Pb}$ are studied through a subtraction procedure to get rid of the correlation peak $(\Delta \phi \sim 0, \Delta \eta \sim 0)$, for pairs of particles originating from the same jet, and of the elongated structure at $\Delta \phi \sim \pi$ for pairs of particles back to back in azimuth, both visible in Fig. 2 

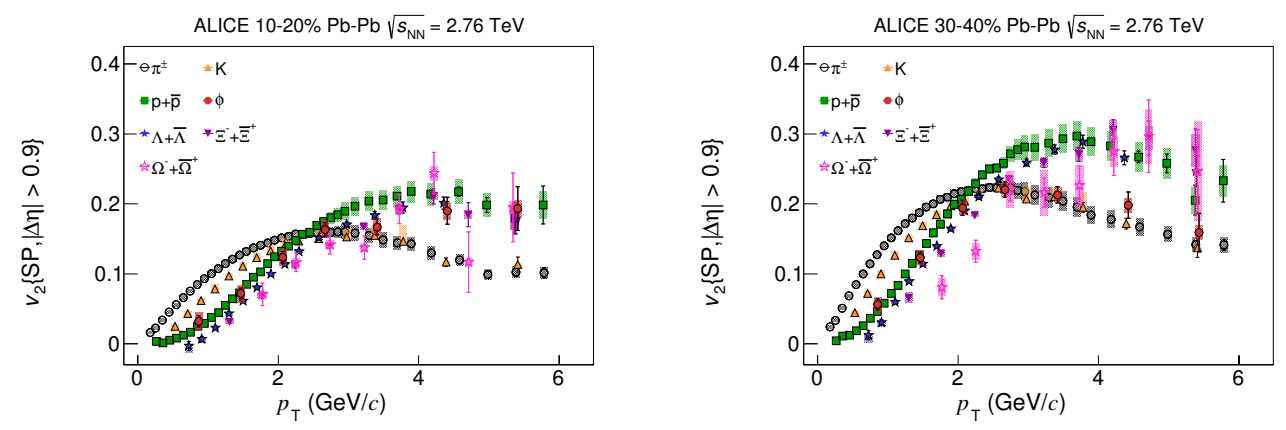

Figure 1. The elliptic flow coefficient $v_{2}$ for different particle species, shown as a function of $p_{\mathrm{T}}$ in $10-20 \%$ (left) and $30-40 \%$ (right) most central $\mathrm{Pb}-\mathrm{Pb}$ collisions [3].

(left and center). Since these structures are clearly visible in the high multiplicity events (0-20\%) and are present, even if less pronounced, also in the low multiplicity classes (60-100\%), we quantify the change from low to high multiplicity event classes by subtracting the latter distribution from the former. The resulting two-particles correlations reveal two long-range ridge structures along the $\Delta \eta$ axis, one centered at $\Delta \phi \sim 0$ (near-side), the other one at $\Delta \phi \sim \pi$ (away-side). Such double-ridge structures were already observed in $\mathrm{Pb}-\mathrm{Pb}$ collisions at the LHC and interpreted in terms of collective expansion. In $\mathrm{p}-\mathrm{Pb}$ collisions their interpretation is still highly debated and possible explanations ranges from collective phenomena in the final state to initial state effects described in a Color Glass Condensate approach. It can be noted that these phenomena were unexpected in $\mathrm{p}-\mathrm{Pb}$ interactions, where the size of the colliding region is too small to allow the development of significant collective effects.
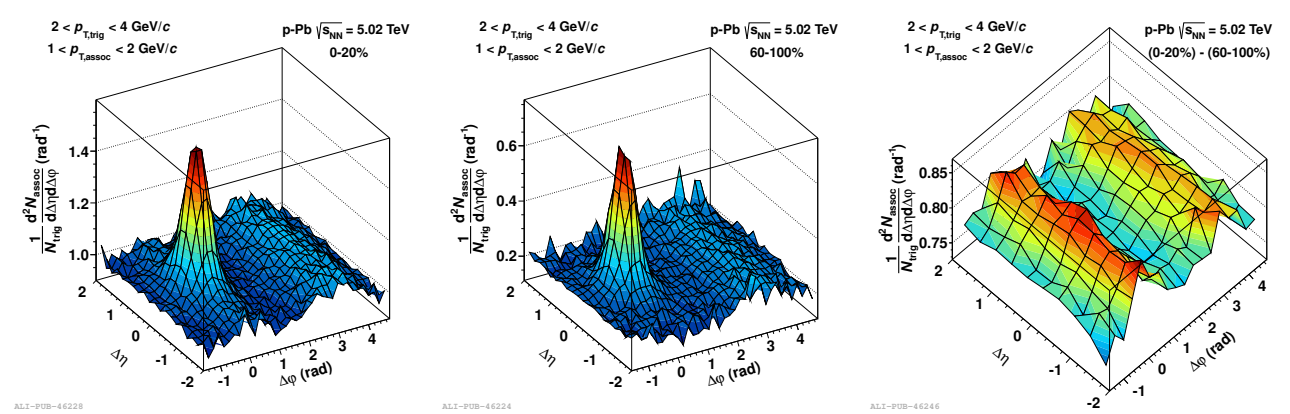

Figure 2. Associated yield per trigger particle in $\Delta \phi$ and $\Delta \eta$ for pairs of charged particles with $2<p_{\mathrm{T}, \text { trig }}<4$ $\mathrm{GeV} / c$ and $1<p_{\mathrm{T}, \text { assoc }}<2 \mathrm{GeV} / c$ in $\mathrm{p}-\mathrm{Pb}$ collisions at $\sqrt{s_{\mathrm{NN}}}=5.02 \mathrm{TeV}$ for the $0-20 \%$ multiplicity class (left), in the $60-100 \%$ event class (center) and the $0-20 \%$ after subtraction of the associated yield obtained in the $60-100 \%$ event class (right) $[4,5]$. 


\section{Hard probes}

\subsection{High- $p_{\mathrm{T}}$ particles and jets}

One of the tools to study the properties of QGP is provided by hard scattering processes of the partonic constituents of the colliding nucleons. These hard scatterings occur early in the collision, so that the outgoing partons travel through the hot expanding medium, finally fragmenting into jets of hadrons. Jet-fragmentation is, therefore, expected to be affected by parton-medium interactions, e.g. radiative and collisional parton energy loss (jet-quenching). Jets are reconstructed grouping particles in a given angular region, within a cone of radius $R$. The modification of the yields is evaluated through the nuclear modification factor $R_{\mathrm{AA}}$, i.e. the ratio of the yields measured in $\mathrm{Pb}-\mathrm{Pb}$ compared to the ones measured in pp collisions, scaled with the number of binary nucleon-nucleon interactions. A value of $R_{\mathrm{AA}}$ different from unity indicates a yield modification induced by the medium, while the $R_{\mathrm{AA}}$ equal to unity indicates that the A-A collisions can be considered as a superposition of binary-scaled nucleon-nucleon interactions. The $R_{\mathrm{AA}}$ for fully reconstructed jets within $\mathrm{R}=0.2$ indicated a strong suppression of the jets yields, in particular in the most central collisions (Fig. 3 (left)) [8].

A similar study based on $\mathrm{p}-\mathrm{Pb}$ collisions shows that the $R_{\mathrm{pA}}$ of charged jets is compatible with unity over a large transverse momentum range (Fig. 3 (right)). This observation confirms that the suppression of high-energy jets in $\mathrm{Pb}-\mathrm{Pb}$ collisions is not due to cold nuclear matter effects (CNM) [9].

Another way to investigate the created medium is based on the high- $p_{\mathrm{T}}$ particles originating from energetic partons in the early stages of the collisions. As previously discussed, parton energy loss in the hot QCD matter is expected to lead to a modification of the yields of the particles originating from partons interacting with the medium. In Fig. 4 (left) the $R_{\mathrm{AA}}$ of high- $p_{\mathrm{T}}$ particles shows a strong suppression increasing with centrality [7]. On the contrary, the pattern observed in $\mathrm{p}-\mathrm{Pb}$ collisions is, also in this case, consistent with unity at high $p_{\mathrm{T}}$ suggesting that no nuclear effects are playing a role. The suppression observed in $\mathrm{Pb}-\mathrm{Pb}$ collisions is, therefore, not an initial-state effect, but it is induced by the hot created medium.
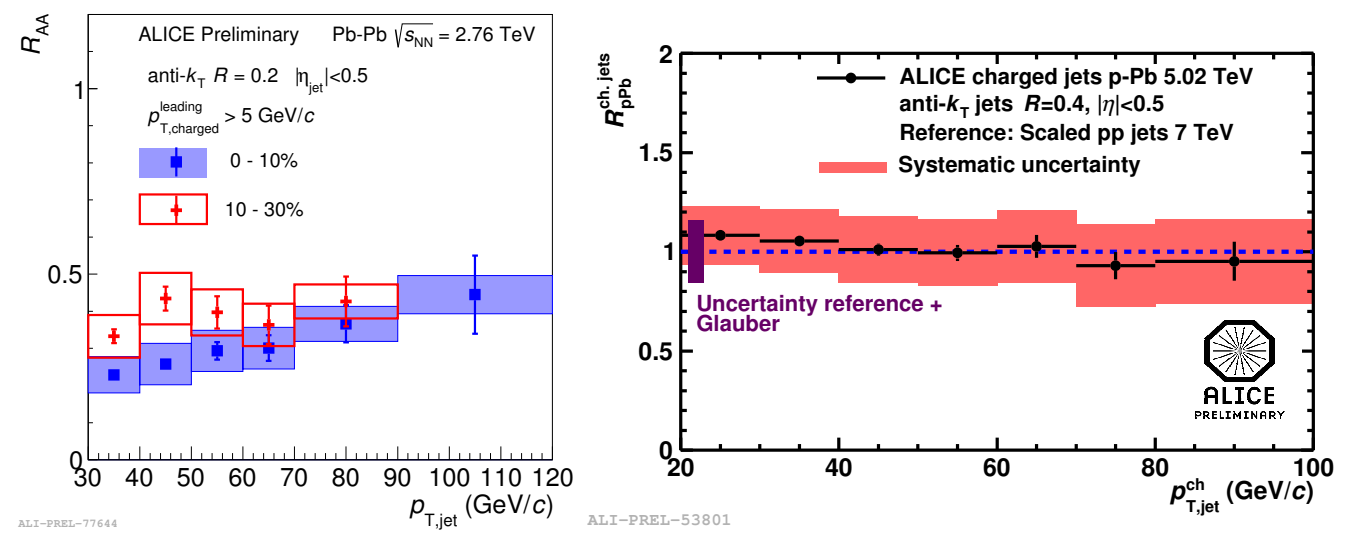

Figure 3. Left: $R_{\mathrm{AA}}$ for fully reconstructed $\mathrm{R}=0.2$ jets with a high $p_{\mathrm{T}}$ track $>5 \mathrm{GeV}$ in $0-10 \%$ and $10-30 \%$ most central events. Right: Nuclear modification factor $R_{\mathrm{pA}}$ for charged jets in p-Pb collisions at $\sqrt{s_{\mathrm{NN}}}=5.02 \mathrm{TeV}$.

Recent ALICE studies have been performed to investigate the centrality dependence of various observables also in $\mathrm{p}-\mathrm{Pb}$ collisions. The division of the events into centrality classes is not straight- 
forward in $\mathrm{p}-\mathrm{Pb}$ collisions, since several biases occur due to the rather small number of nucleons participating in the collisions, leading to a large influence of the fluctuations. The centrality selection is, therefore, more difficult in these collisions, with respect to the $\mathrm{Pb}-\mathrm{Pb}$ case. In order to minimize the effect of the aforementioned kinematic biases, ALICE has developed an hybrid approach. In this method, events are sliced into classes based on the signals released in the zero-degree neutron calorimeters. The average number of nucleon-nucleon collisions in a given class is obtained by assuming a scaling of the multiplicity with, for example, the number of nucleons participating in the collision. The nuclear modification factor is, in this case, called $Q_{\mathrm{pPb}}$, to underline the existence of a centrality-bias in the definition of the event activity classes. As an example, in Fig. 4 (right) the $Q_{\mathrm{pPb}}$ of the charged particles is shown, in various event activity classes, as a function of $p_{\mathrm{T}}$. The result indicates that the $Q_{\mathrm{pPb}}$ of the high- $p_{\mathrm{T}}$ charged particles is flat, i.e. high momentum particles do not exhibits the suppression that in $\mathrm{Pb}-\mathrm{Pb}$ is attribuited to interactions with the medium. The observed flatness is independent of the activity class.
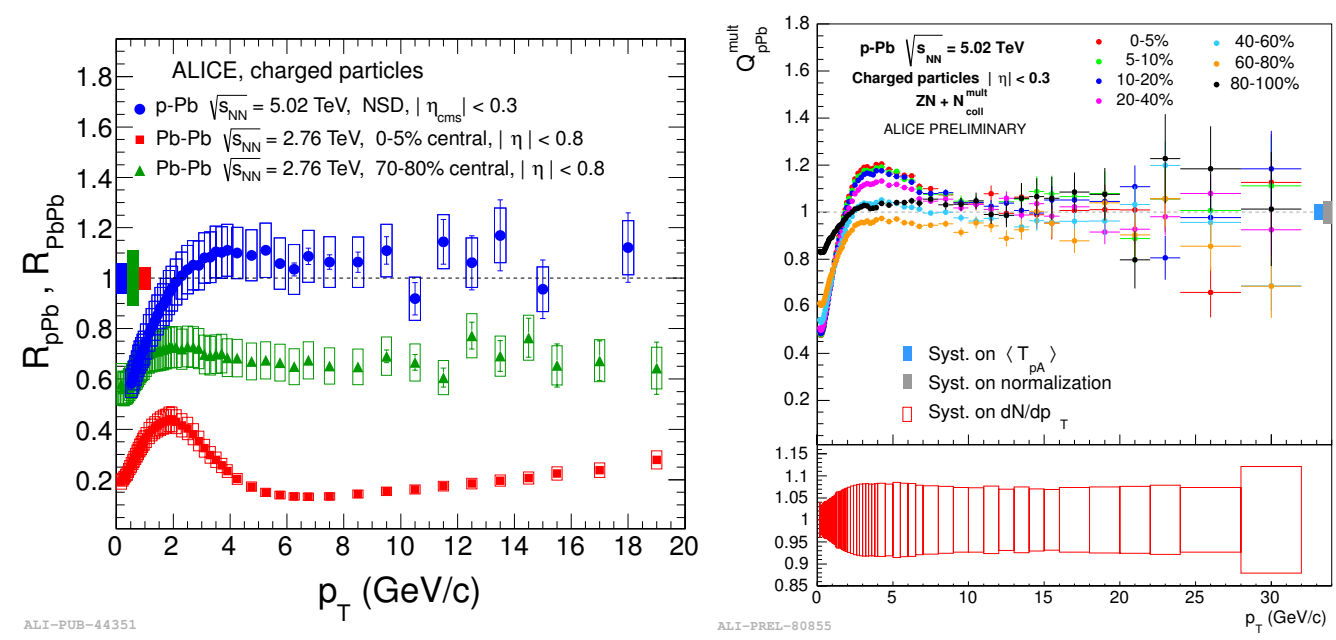

Figure 4. Left: The nuclear modification factor of charged particles as a function of transverse momentum in NSD p-Pb collisions at $\sqrt{s_{\mathrm{NN}}}=5.02 \mathrm{TeV}$. Data are compared to measurements in central $(0-5 \%)$ and peripheral (70-80\%) $\mathrm{Pb}-\mathrm{Pb}$ collisions at $\sqrt{s_{\mathrm{NN}}}=2.76 \mathrm{TeV}$ [7]. Right: $Q_{\mathrm{pPb}}$ evaluated with the hybrid approach, assuming that charged particle multiplicity at midrapidity scales with the number of participating nucleons.

\subsection{Heavy flavours}

Heavy quarks such as charm and beauty are produced in hard parton scattering processes in the initial stage of the hadronic collisions. Being produced in the early stages of the fireball evolution, the production of heavy-flavours is an interesting tool to probe the characteristics of the partonic medium. Theoretical energy loss models predict a hierarchical dependence on the color-charge and mass of the involved partons, leading to a increasing energy loss going from beauty, to charm and eventually to gluons. It is, therefore, interesting to compare the nuclear modification factors of light hadrons to the one of $\mathrm{D}$ and $\mathrm{B}$ mesons. The $R_{\mathrm{AA}}$ of $\mathrm{D}$ mesons is shown in Fig. 5 (left) in two $\mathrm{Pb}-\mathrm{Pb}$ centrality classes $(0-20 \%$ and $40-80 \%)$ [6]. A clear suppression towards high $p_{\mathrm{T}}$ is observed, and this effect is stronger 
in the most central collisions. The $\mathrm{D}$ meson $R_{\mathrm{pA}}$ has also been measured in $\mathrm{p}-\mathrm{Pb}$ collisions and it shows no suppression as a function of $p_{\mathrm{T}}$, suggesting that the suppression observed in $\mathrm{Pb}-\mathrm{Pb}$ interactions is related to effects induced by the hot partonic matter [10]. The $R_{\mathrm{AA}}$ of $\mathrm{D}$ mesons in $\mathrm{Pb}-\mathrm{Pb}$ is similar, within uncertainties to the pion one, but more statistics is needed to draw conclusions on the role of the color charge dependence of energy loss. In a similar way, as shown in Fig. 5 (right) the D meson $R_{\mathrm{AA}}$ is compared to the CMS result for non-prompt $\mathrm{J} / \psi$ coming from B decays [11]. Even if a detailed comparison is not straightforward, given the different rapidity range, there is an indication for a stronger suppression for mesons including $c$ quarks, in agreement with theoretical expectations.
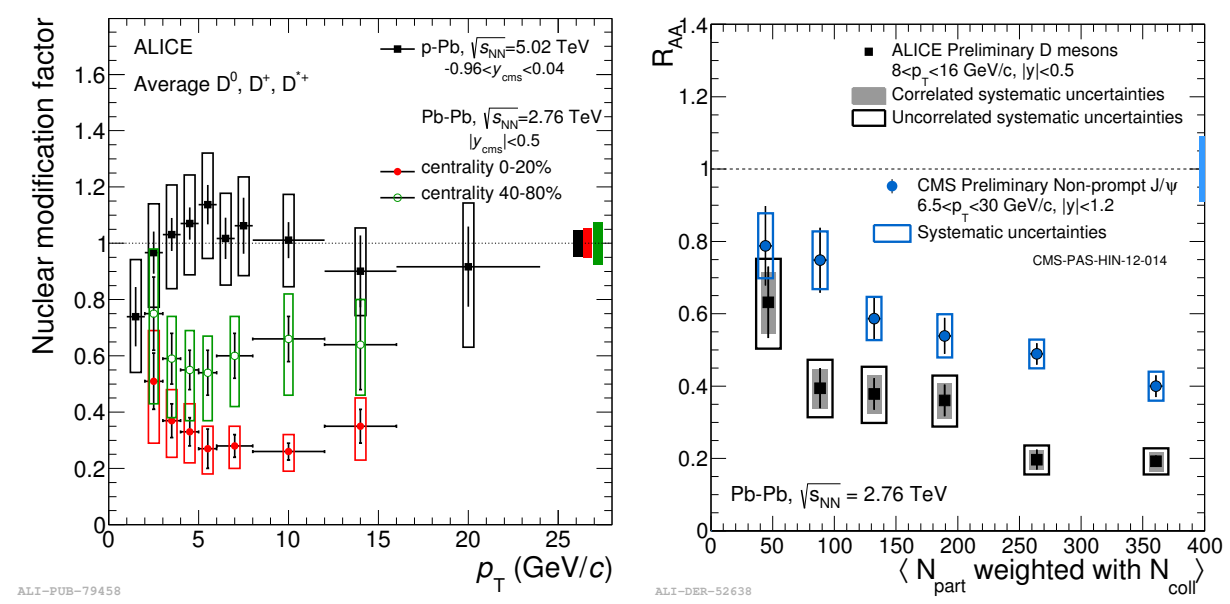

Figure 5. Left: $R_{\mathrm{pPb}}$ of the average of $\mathrm{D}^{0}, \mathrm{D}^{+}$and $\mathrm{D}^{-}$mesons as a function of $p_{\mathrm{T}}$, compared to the $\mathrm{D}$ meson $R_{\mathrm{AA}}$ in the $20 \%$ and in the $40-80 \% \mathrm{~Pb}-\mathrm{Pb}$ collisions at $\sqrt{s_{\mathrm{NN}}}=2.76 \mathrm{TeV}$ [10]. Right: the $\mathrm{D}$ meson $R_{\mathrm{AA}}$ compared to the CMS result for the $\mathrm{J} / \psi$ from $\mathrm{B}$ decays [11], as a function of the centrality of the collisions.

\subsection{Quarkonia}

The high density of colour charges, present in a plasma of quarks and gluons (QGP) created in heavyion collisions, is expected to screen the binding between heavy (charm or beauty) $Q$ and $\bar{Q}$ quarks, suppressing the production of quarkonium states with respect to the production in $\mathrm{pp}$ [12].

The ALICE Collaboration has studied both the $\mathrm{J} / \psi$ and the more loosely bound $\psi(2 \mathrm{~S})$ production in $\mathrm{Pb}-\mathrm{Pb}$ and $\mathrm{p}-\mathrm{Pb}$ collisions $[13,14]$. Charmonium resonances are measured, through their dielectron or dimuon decay channel, at mid-rapidity or at forward rapidity respectively [15]. The $\mathrm{J} / \psi R_{\mathrm{AA}}$, measured in $\mathrm{Pb}-\mathrm{Pb}$ collisions shows, in both rapidity regions, a very strong suppression, with almost no centrality dependence above about 100 participant nucleons. The result shows a stricking difference with respect the the pattern observed at RHIC energies, since the $\mathrm{J} / \psi$ suppression measured by ALICE is weaker with respect to the one evaluated at lower energies (Fig. 6 (left)). Given the hotter and denser medium created at LHC, one would have expected a stronger suppression at higher energies. Therefore, to interpret the observed behaviour a new production mechanism has to be invoked. Due to the increase of the $c \bar{c}$ production cross-section with the collision energy, at LHC J/ $\psi$ can be produced by "(re)combination" at the hadronization phase or during the fireball evolution. Being related to the abundance of charm quarks, this new mechanism was playing a negligible role at lower energies, 
while at the LHC energies it might counterbalance the $\mathrm{J} / \psi$ suppression [16]. As expected by theory predictions, the $\mathrm{J} / \psi$ produced by (re)combination dominate at low transverse momentum, while their contribution becomes negligible at high $p_{\mathrm{T}}$.
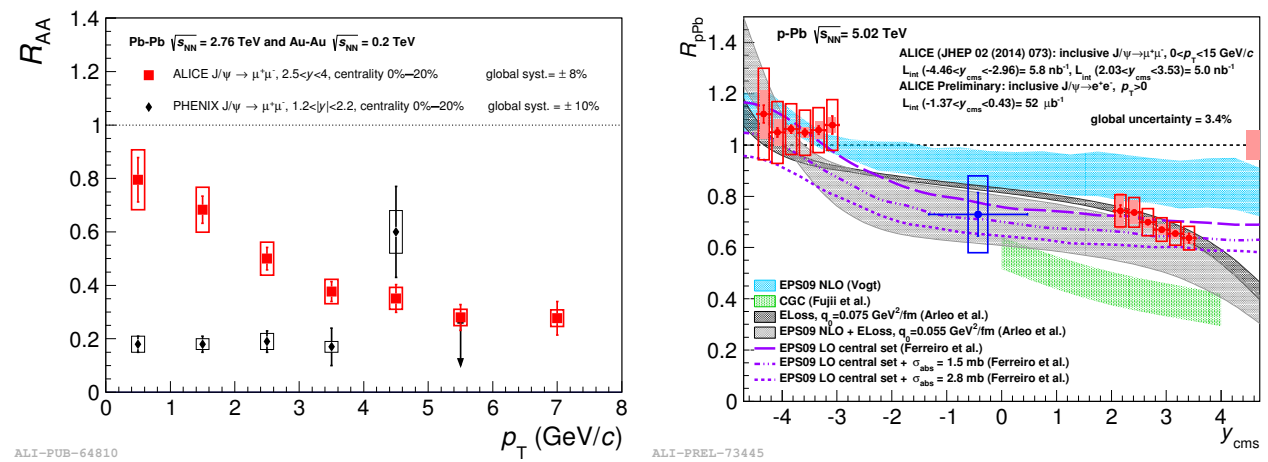

Figure 6. Left: $\mathrm{J} / \psi$ nuclear modification factor as a function of $p_{\mathrm{T}}$, compared to the PHENIX result [13]. Right: $\mathrm{p}-\mathrm{Pb} \mathrm{J} / \psi$ nuclear modification factor as a function of rapidity, compared to theoretical calculations including cold nuclear matter effects as nuclear parton shadowing, energy loss and nuclear absorption cross section [14].

The most recent ALICE results on quarkonium have been obtained in $\mathrm{p}-\mathrm{Pb}$ collisions [14]. The $\mathrm{J} / \psi$ production is strongly modified already in this collision system due to the presence of the socalled cold nuclear matter effects as parton shadowing and energy loss (Fig 6 (right)). Since these effects are present also in $\mathrm{Pb}-\mathrm{Pb}$ interactions, the $R_{\mathrm{pA}}$ result can be considered as a useful tool to help understanding the contribution of cold nuclear matter to the suppression of the $\mathrm{J} / \psi$ yields observed in $\mathrm{Pb}-\mathrm{Pb}$. Under the assumptions that i) nuclear shadowing is the dominanting cold nuclear matter effect affecting $\mathrm{J} / \psi$ production, ii) its effect on the two colliding $\mathrm{Pb}$ nuclei can be factorized, the product $R_{\mathrm{pPb}} \times R_{\mathrm{Pbp}}$ can be considered as an estimate of CNM effects in $\mathrm{Pb}-\mathrm{Pb}$ collisions. Comparing the extrapolation of $\mathrm{p}-\mathrm{Pb}$ results to $\mathrm{Pb}-\mathrm{Pb}$ collisions, we can notice that the suppression observed in $\mathrm{Pb}-\mathrm{Pb}$ collisions exceed the cold nuclear matter expectations, based on the $R_{\mathrm{pA}}$ results (Fig. 7 (left)). This behaviour confirms that hot matter effects are dominant in $\mathrm{Pb}-\mathrm{Pb}$ interactions.

Finally, in $\mathrm{p}-\mathrm{Pb}$ interactions, the $\mathrm{J} / \psi$ and $\psi(2 \mathrm{~S})$ behaviour has been investigated also as a function of the event activity. The $\mathrm{J} / \psi Q_{\mathrm{pPb}}$ shows a suppression, in the p-going direction, increasing with the event activity, while no significant suppression is observed in the Pb-going direction. These findings are consistent with the expectations based on the aforementioned cold nuclear matter effects. The $\psi(2 \mathrm{~S})$ shows a similar behaviour to the $\mathrm{J} / \psi$ one in the p-going direction. In the $\mathrm{Pb}$-going one, however, contrary to the $\mathrm{J} / \psi$, the $\psi(2 \mathrm{~S})$ is suppressed, as shown in Fig. 7 (right). Since the expectations based on cold nuclear matter effects should be similar for both resonances, the observed unexpected different behaviour suggests that additional final state effects should affect the observed yields.

\section{Conclusions}

The ALICE Collaboration has deeply investigated the properties of the hot medium created in $\mathrm{Pb}-\mathrm{Pb}$ collisions. In particular, the most recent results have now improved the precision reached in the first measurements in the various areas. For example, a step further in the understanding of the collective behaviour has been done measuring the identified particle ellliptic flow. Results indicate a clear mass 

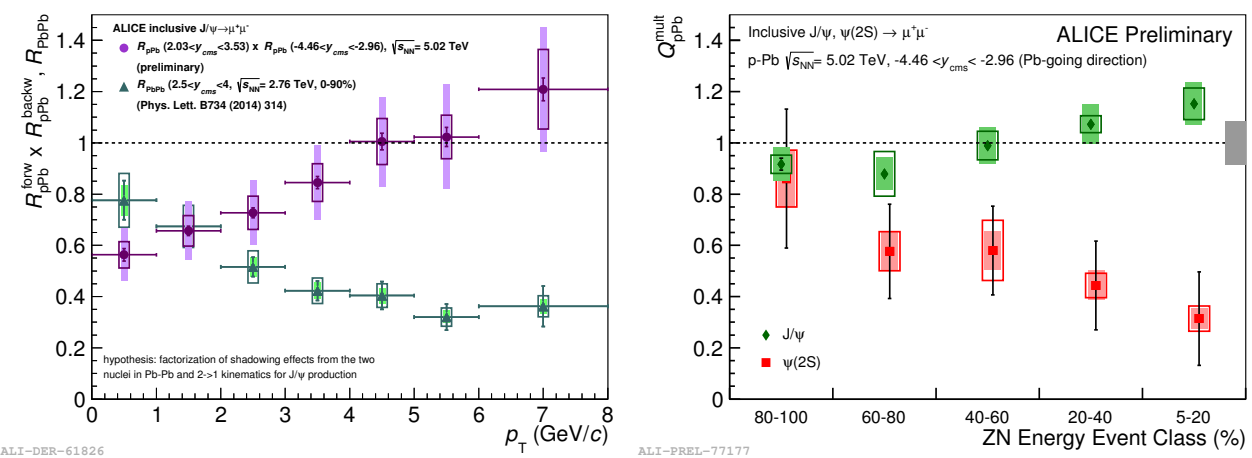

Figure 7. Left: The $\mathrm{J} / \psi R_{\mathrm{AA}}$, as a function of $p_{\mathrm{T}}$, compared to the cold nuclear matter expectations based on the $R_{\mathrm{pA}}$ results. Right: $\mathrm{J} / \psi$ and $\psi(2 \mathrm{~S}) Q_{\mathrm{pPb}}$ versus event activity, in the $\mathrm{Pb}$-going direction

ordering for light and strange hadrons for $p_{\mathrm{T}}<2.5 \mathrm{GeV} / \mathrm{c}$, while spectra and $v_{2}$ measurements of the $\phi$ meson suggest that the mass (and not the number of constituent quarks) drives the shape and the size of the elliptic flow in most central collisions. Recently new results obtained in $\mathrm{p}-\mathrm{Pb}$ collisions have been presented. While for several observables $\mathrm{p}-\mathrm{Pb}$ collisions turn out to be a superposition on pp collisions, other measurements point to an unexpected behaviour potentially related to a collective origin. Furthermore, the unexpected stronger suppression for the $\psi(2 \mathrm{~S})$ with respect to the $\mathrm{J} / \psi$ suggests the presence of final state effects setting in in $\mathrm{p}-\mathrm{Pb}$ interactions. These new findings still need a well assessed theoretical explanation. This large wealth of data will be soon complemented by the results expected from the incoming LHC Run-II, where the higher collision energies foreseen will allow to sharpen the scenario outlined from the first LHC Run.

\section{References}

[1] K. Aamodt et al. (ALICE Collaboration), JINST 3 (2008) S08002.

[2] K. Aamodt et al. (ALICE Collaboration), Phys. Lett. B696 (2010) 328.

[3] B. Abelev et al. (ALICE Collaboration), arXiv:1405.4632.

[4] B. Abelev et al. (ALICE Collaboration), Phys. Lett. B719 (2013) 29.

[5] B. Abelev et al. (ALICE Collaboration), Phys. Lett. B726 (2013) 164.

[6] B. Abelev et al. (ALICE Collaboration), JHEP 1209 (2012) 112.

[7] B. Abelev et al. (ALICE Collaboration), Phys. Rev. Lett. 110 (2013) 082302.

[8] B. Abelev et al. (ALICE Collaboration), JHEP 30 (2014) 13.

[9] B. Abelev et al. (ALICE Collaboration), arXiv:1405.3452.

[10] B. Abelev et al. (ALICE Collaboration), arXiv:1310.3612.

[11] S. Chatrchyan et al. (CMS Collaboration) JHEP 1205 (2012) 063, CMS-PAS-HIN-12-014.

[12] T. Matsui and H. Satz, Phys. Lett. B178 (1986) 416.

[13] B. Abelev et al. (ALICE Collaboration), arXiv:1405.3796 (submitted to JHEP).

[14] B. Abelev et al. (ALICE Collaboration), JHEP 1402 (2014) 073.

[15] I. Das et al. (ALICE Collaboration), these proceedings.

[16] B. Abelev et al. (ALICE Collaboration) Phys. Lett. B743 (2014) 314. 